\title{
Neutrino electromagnetic properties: a window to new physics - II
}

\author{
Alexander Studenikin*ab \\ ${ }^{a}$ Department of Theoretical Physics, Moscow State University \\ 119992 Moscow, Russia \\ ${ }^{b}$ Joint Institute for Nuclear Research \\ Dubna 141980, Moscow Region, Russia \\ E-mail: studenikesrd.sinp.msu.ru
}

\begin{abstract}
There is merely a short note on the selected issues of neutrino electromagnetic properties with focus on effects of new physics. The meaning of "new physics" is twofold: 1) a massive neutrino have nonzero electromagnetic properties that can be considered as manifestation of new physics beyond the Standard Model, and 2) in studies of neutrinos electromagnetic interactions new effects are predicted that can lead to new phenomena accessible for observations.
\end{abstract}

The European Physical Society Conference on High Energy Physics

5-12 July, 2017

Venice

${ }^{*}$ Speaker. 


\section{Electromagnetic properties of neutrino}

After the Nobel Prize of 2015 in physics was awarded to Arthur McDonald and Takaaki Kajita for the discovery of neutrino oscillations, there can be no doubt that neutrinos are massive particles. It has been know since quite many years [四] that massive neutrinos should have nonzero magnetic moments. Although up to now there are no indications in favour of nonzero neutrino electromagnetic properties, neither from terrestrial experiments nor from astrophysical observations, the electromagnetic properties is one of popular issues related to neutrinos and this problem has been discussed many times in recent literature (see, for instance, [ㅁ]-[可 ). A complete review on neutrino electromagnetic properties and neutrino electormagnetic interactions is given in [ए0]. In [1], [2] an addendum to previous reviews are provided and the most recent new aspects and prospects related to neutrino electromagnetic interactions that have appeared after publication of the review paper [ए]] are discussed.

It has been often claimed (see, for instance, [ [ד]) that neutrino electromagnetic properties open a window to new physics. In this short note we would like to justify this statement and to focus a discussion on to what extend and what kind of new physics neutrino electromagnetic properties do communicate with.

\section{Neutrino magnetic moment}

Consider the magnetic moment as the most well theoretically appreciated and experimentally studied (constrained) electromagnetic characteristic of neutrinos. Within the initial formulation of the Standard Model neutrinos are massless particles with zero magnetic moment. Thus, the would be nonzero neutrino magnetic moment regardless of its value should indicate the existence of new physics beyond the Standard Model. Indeed, as it has been shown in [四] a minimal extension of the Standard Model with right-handed neutrinos yields for the diagonal magnetic moment of a Dirac neutrino to be proportional to the neutrino mass $m_{i}$,

$$
\mu_{i i}^{D}=\frac{3 e G_{F} m_{i}}{8 \sqrt{2} \pi^{2}} \approx 3.2 \times 10^{-19}\left(\frac{m_{i}}{1 \mathrm{eV}}\right) \mu_{B},
$$

where $\mu_{B}$ is the Bohr magneton. For Majorana neutrinos the diagonal magnetic moments are zero in the neutrino mass basis and only transition (off-diagonal) magnetic moments $\mu_{i j}^{M}(i \neq j)$ can be nonzero in this case.

3. The value of neutrino magnetic moment (2. present experimental limits if to account for the existed constraints on neutrino masses. Note that in general transition magnetic moments are even smaller due to the GIM cancelation mechanism.

The best laboratory upper limit on neutrino magnetic moment has been obtained by the GEMMA collaboration that investigates the reactor antineutrino-electron scattering at the Kalinin Nuclear Power Plant (Russia) [[13]. Within the presently reached electron recoil energy threshold of $T \sim 2.8$ $\mathrm{keV}$ the neutrino magnetic moment is bounded from above by the value

$$
\mu_{v}<2.9 \times 10^{-11} \mu_{B}(90 \% \text { C.L. }) .
$$

This limit, obtained from unobservant distortions in the recoil electron energy spectra, is valid for both Dirac and Majorana neutrinos and for both diagonal and transition moments. The most recent 
stringent constraint on the electron effective magnetic moment

$$
\mu_{v_{e}} \leq 2.8 \times 10^{-11} \mu_{B}
$$

has been reported by the Borexino Collaboration [14]].

A strict astrophysical bound on the neutrino magnetic moment is provided by the observed properties of globular cluster stars and amounts to [प5]] (see also [ㅁ, [प]])

$$
\left(\sum_{i, j}\left|\mu_{i j}\right|^{2}\right)^{1 / 2} \leq(2.2-2.6) \times 10^{-11} \mu_{B}
$$

This stringent astrophysical constraint on neutrino magnetic moments is applicable to both Dirac and Majorana neutrinos.

There is a huge gap of many orders of magnitude between the present experimental limits on neutrino magnetic moments and the prediction of a minimal extension of the Standard Model. Therefore, if any direct experimental confirmation of nonzero neutrino magnetic moment were obtained in a reasonable future, it would open a window to new physics beyond a minimal extension of the Standard Model.

Much larger values for a neutrino magnetic moments are predicted in different other extensions of the Standard Model. However, there is a general problem for a theoretical model of how to get large magnetic moment for a neutrino and simultaneously to avoid an unacceptable large contribution to the neutrino mass (see the corresponding discussion in [ए0] and references therein). If a contribution to the neutrino magnetic moment of an order $\mu_{v} \sim \frac{e G}{\Lambda}$ is generated by physics beyond a minimal extension of the Standard Model at an energy scale characterized by $\Lambda$, then the corresponding contribution to the neutrino mass is $\delta m_{v} \sim \frac{\Lambda^{2}}{2 m_{e}} \frac{\mu_{v}}{\mu_{B}}=\frac{\mu_{v}}{10^{-18} \mu_{B}}\left(\frac{\Lambda}{1 T e v}\right)^{2} \mathrm{eV}$. Therefore, a particular fine tuning is needed to get large value for a neutrino magnetic moment while keeping the neutrino mass within experimental bounds. Different possibilities to have large magnetic moment for a neutrino were considered in the literature (see in [0]]).

A general and termed model-independent upper bound on the Dirac neutrino magnetic moment, that can be generated by an effective theory beyond a minimal extension of the Standard Model, has been derived in [ए8]]: $\mu_{v} \leq 10^{-14} \mu_{B}$. Note that the corresponding limit for transition moments of Majorana neutrinos is much weaker [19]. Thus, the value of a neutrino magnetic moment once observed experimentally at the level not less than $\mu_{v} \sim 10^{-14} \mu_{B}$ would provide information on the nature of neutrinos. This can be also considered as a view on the realm of new physics.

\section{Neutrino electric moment}

From the most general form of the neutrino electromagnetic vertex function $\Lambda_{i j}\left(q^{2}\right)$ (see for detailed discussion [ए]]) there are three other sets (in addition to the magnetic moments $\mu_{i j}$ ) of electromagnetic characteristics that determine a neutrino coupling with real photons $\left(q^{2}=0\right)$. They are namely the dipole electric moments $\varepsilon_{i j}$, anapole moments $a_{i j}$ and millicharges $q_{i j}$. In the theoretical framework with $C P$ violation a neutrino can have nonzero electric moments $\varepsilon_{i j}$. In the laboratory neutrino scattering experiments for searching the neutrino magnetic moment (like, for 
instance, the mentioned above GEMMA experiment) the electric moment contributions interfere with those due to magnetic moments. Thus, these kind of experiments also provide constraints on

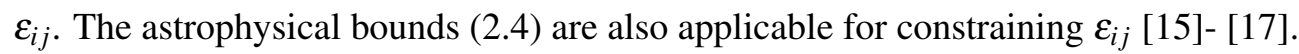

\section{Neutrino electric millicharge}

There are extensions of the Standard Model that allow for nonzero neutrino electric millicharges. This option can be provided by not excluded experimentally possibilities for hypercharhge dequantization or another new physics related with an additional $U(1)$ symmetry peculiar for extended theoretical frameworks. Neutrino millicharges are strongly constrained on the level $q_{v} \sim 10^{-21} e_{0}\left(e_{0}\right.$ is the value of an electron charge) from neutrality of the hydrogen atom.

A nonzero neutrino millicharge $q_{v}$ would contribute to the neutrino electron scattering in the terrestrial experiments. Therefore, it is possible to get bounds on $q_{v}$ in the reactor antineutrino experiments GEMMA. The most stringent constraint using the GEMMA data is $q_{v} \leq 1.5 \times 10^{-11} e_{0}$ [20] (see also [2] ]

A neutrino millicharge might have specific phenomenological consequences in astrophysics because of new electromagnetic processes are opened due to a nonzero charge. Following this line, the most stringent astrophysical constraint on neutrino millicharges $q_{v} \leq 1.3 \times 10^{-19} e_{0}$ was obtained in [22]. This bound follows from the impact of the neutrino star turning mechanism (STV) [D2] that can be charged as a new physics phenomenon end up with a pulsar rotation frequency shift engendered by the motion of escaping from the star neutrinos on curved trajectories due to millicharge interaction with a constant magnetic field.

\section{Neutrino charge radius}

Even if a neutrino millicharge is vanishing, the electric form factor $f_{i j}^{q}\left(q^{2}\right)$ can still contain nontrivial information about neutrino electromagnetic properties. The corresponding electromagnetic characteristics is determined by the derivative of $f_{i j}^{q}\left(q^{2}\right)$ over $q^{2}$ at $q^{2}=0$ and is termed neutrino charge radius, $r_{i j}^{2}=-\left.6 \frac{d f_{i j}^{q}\left(q^{2}\right)}{d q^{2}}\right|_{q^{2}=0}$. A neutrino charge radius (that is indeed the charges radius squared) contributes to the neutrino scattering cross section on electrons and thus can be constrained by the corresponding laboratory experiments [223]. In all (see, for instance, [ए人]) but one previous studies it was claimed that the effect of the neutrino charge radius can be included just as a shift of the vector coupling constant $g_{V}$ in the weak contribution to the cross section. However, as it has been recently illustrated, in [24] within the direct calculations of the cross section accounting for all possible neutrino electromagnetic characteristics and neutrino mixing, this is not the fact. The neutrino charge radius dependence of the cross section indeed is more complicated and there is, in particular, the dependence on the interference terms of the type $g_{V} r_{i j}^{2}$ that can't be obtained just only by the corresponding shift of the constant $g_{V}$.

\section{Neutrino spin precession in magnetic field}

One of an important phenomenon among several processes of neutrino electromagnetic interacts is neutrino spin and spin-flavour precession in magnetic fields. The origin of these effects is the 
neutrino magnetic moment interaction with a transversal magnetic field determined by $\mu_{v} B_{\perp}$. The neutrino spin precession in a transverse magnetic field can result in the neutrino helicity flip that can have important phenomenological consequences because an active neutrino $v_{L}$ can be converted to a sterile one $v_{R}$ in invironments with a magnetic field. The precession $v_{e L} \rightarrow v_{e R}$ in the magnetic field of the Sun was first considered in [25], a similar effect in magnetic fields of supernovae and neutron stars came into sight for the first time in [山].

\section{Neutrino spin precession in transversal matter current}

There is a phenomenon of new physics related to the neutrino spin precession in magnetic fields. For many years, until 2004, it was believed that a neutrino helicity precession and the corresponding spin oscillations can be induced by the neutrino magnetic interactions with the transversal magnetic field. A new and very interesting possibility for neutrino spin (and spin-flavour) oscillations engendered by the neutrino interaction with matter background was proposed and investigated in [26]. It was shown that neutrino spin oscillations can be induced not only by the neutrino interaction with a magnetic field but also by neutrino interactions with matter in the case when there is a transversal matter current (or a transversal matter matter polarization). The is no need for neutrino magnetic moment interaction in this case. The origin of the oscillations $v_{L} \Leftrightarrow v_{R}$ in the transversal matter currents $j_{\perp}$ is the neutrino weak interactions with moving matter and the corresponding mixing between neutrino states $v_{L}$ and $v_{R}$ is determined by $G_{F} j_{\perp}$. This new effect has been explicitly highlighted in [26, [27], recently the existence of this effect was confirmed in [28]]. For historical notes reviewing studies and the detailed derivation of the discussed effect see [ए], [2] and [20].

\section{Conclusions and future prospects}

The foreseen progress in constraining neutrino electromagnetic characteristics is related, first of all, with the expected new results from the GEMMA experiment measurements of the reactor antineutrino cross section on electrons at Kalinin Power Plant. The new set of data is expected to arrive next year. The electron energy threshold will be as low as $350 \mathrm{eV}$ ( or even lower, $\sim 200 \mathrm{eV}$ ). This will provide possibility to test the neutrino magnetic moment on the level of $\mu_{v} \sim 0.9 \times$ $10^{-12} \mu_{B}$ and also to test the millicharge on the level of $q_{v} \sim 1.8 \times 10^{-13} e_{0}$ [LO]. For the next future, presently it seems unclear whether further progress in constraining the neutrino electromagnetic characteristics would be achievable with this type of the reactor antineutrino experiment. In this concern, a rather promising claim was made in [30, [3]]. It was shown that even much smaller values of the Majorana neutrino transition moments would probably be tested in future high-precision experiments with the astrophysical neutrinos. In particular, observations of supernova fluxes in the JUNO experiment (see [B2]- [B4] ) may reveal the effect of collective spin-flavour oscillations due to the Majorana neutrino transition moment $\mu_{v}^{M} \sim 10^{-21} \mu_{B}$.

To conclude, the existing current constraints on the flavour neutrino charge radius $r_{e, \mu, \tau}^{2} \leq$ $10^{-32}-10^{-31} \mathrm{~cm}^{2}$ from the scattering experiments differ only by 1 to 2 orders of magnitude from the values $r_{e, \mu, \tau}^{2} \leq 10^{-33} \mathrm{~cm}^{2}$ calculated within the minimally extended Standard Model with righthanded neutrinos [리 $]$. This indicates that the minimally extended Standard Model neutrino charge radii could be experimentally tested in the near future. Note that there is a need to re-estimate 
experimental constraints on $r_{e, \mu, \tau}^{2}$ from the scattering experiments following new derivation of the cross section [24] that properly accounts for the interference of the weak and charge radius electromagnetic interactions and also for the neutrino mixing.

\section{Acknowledgements}

This work was supported by the Russian Foundation for Basic Research under grants No. 1602-01023 A and No. 17-52-53133 GFEN_a.

\section{References}

[1] K. Fujikawa and R. Shrock, The Magnetic Moment of a Massive Neutrino and Neutrino Spin Rotation, Phys. Rev. Lett. 45 (1980) 963.

[2] G. G. Raffelt, Astrophysics probes of particle physics, Phys. Rept. 333 (2000) 593.

[3] M. Nowakowski, E. A. Paschos and J. M. Rodriguez, All electromagnetic form-factors, Eur. J. Phys. 26 (2005) 545.

[4] H. T. Wong and H. B. Li, Neutrino magnetic moments, Mod. Phys. Lett. A 20 (2005) 1103.

[5] A. B. Balantekin, Neutrino magnetic moment, AIP Conf. Proc. 847 (2006) 128.

[6] C. Giunti and A. Studenikin, Neutrino electromagnetic properties, Phys. Atom. Nucl. 72 (2009) 2089.

[7] A. Studenikin, Neutrino magnetic moment: A Window to new physics, Nucl. Phys. Proc. Suppl. 188 (2009) 220.

[8] C. Broggini, C. Giunti and A. Studenikin, Electromagnetic Properties of Neutrinos, Adv. High Energy Phys. 2012 (2012) 459526.

[9] E. Akhmedov, Majorana neutrinos and other Majorana particles:Theory and experiment, arXiv: 1412.3320 [hep-ph].

[10] C. Giunti and A. Studenikin, Neutrino electromagnetic interactions: a window to new physics, Rev. Mod. Phys. 87 (2015) 531.

[11] A. Studenikin, Status and perspectives of neutrino magnetic moments, J. Phys. Conf. Ser. 718 (2016) 062076.

[12] A. Studenikin, Electromagnetic properties of neutrinos: three new phenomena in neutrino spin oscillations, EPJ Web Conf. 125 (2016) 04018.

[13] A. G. Beda, V. B. Brudanin, V. G. Egorov et al., The results of search for the neutrino magnetic moment in GEMMA experiment, Adv. High Energy Phys. 2012 (2012) 350150.

[14] M. Agostini et al. [Borexino Collaboration], Limiting neutrino magnetic moments with Borexino Phase-II solar neutrino data, arXiv:1707.09355 [hep-ex].

[15] G. G. Raffelt, New bound on neutrino dipole moments from globular-cluster stars, Phys. Rev. Lett. 64 (1990) 2856.

[16] N. Viaux, M. Catelan, P. B. Stetson, G. G. Raffelt et al., Particle-physics constraints from the globular cluster M5: neutrino dipole moments, Astron. \& Astrophys. 558 (2013) A12.

[17] S. Arceo-Díaz, K.-P. Schröder, K. Zuber and D. Jack, Constraint on the magnetic dipole moment of neutrinos by the tip-RGB luminosity in $\omega$-Centauri, Astropart. Phys. 70 (2015) 1. 
[18] N. F. Bell, V. Cirigliano, M. J. Ramsey-Musolf et al How magnetic is the Dirac neutrino?, Phys. Rev. Lett. 95 (2005) 151802.

[19] N. F. Bell, M. Gorchtein, M. J. Ramsey-Musolf, P. Vogel and P. Wang, Model independent bounds on magnetic moments of Majorana neutrinos, Phys. Lett. B 642 (2006) 377.

[20] A. Studenikin, New bounds on neutrino electric millicharge from limits on neutrino magnetic moment, Europhys.Lett. 107 (2014) 21001. [arXiv:1302.1168 [hep-ph]].

[21] C. Patrignani et al. [Particle Data Group], Review of Particle Physics, Chin. Phys. C 40 (2016) 100001 .

[22] A. Studenikin and I. Tokarev, Millicharged neutrino with anomalous magnetic moment in rotating magnetized matter, Nucl. Phys. B 884 (2014) 396.

[23] J. Bernabeu, J. Papavassiliou and D. Binosi, The Neutrino charge radius in the presence offermion masses, Nucl. Phys. B 716 (2005) 352.

[24] K. Kouzakov and A. Studenikin, Electromagnetic properties of massive neutrinos in low-energy elastic neutrino-electron scattering, Phys. Rev. D 95 (2017) 055013.

[25] A. Cisneros, Effect of neutrino magnetic moment on solar neutrino observations, Astrophys. Space Sci. 10 (1971) 87.

[26] A. Studenikin, Neutrinos in electromagnetic fields and moving media, Phys. Atom. Nucl. 67 (2004) 993.

[27] A. Studenikin, Neutrino in magnetic fields: From the first studies to the new effects in neutrino oscillations, hep-ph/0407010.

[28] A. Kartavtsev, G. Raffelt, H. Vogel, Neutrino propagation in media: Flavor-, helicity-, and pair correlations, Phys. Rev. D 91 (2015) 125020.

[29] A. Studenikin, Neutrino spin and spin-flavour oscillations in transversally moving or polarized matter, J. Phys. Conf. Ser. 888 (2017) 012221.

[30] A. de Gouvea and S. Shalgar, Effect of transition magnetic moments on collective supernova neutrino oscillations, JCAP 1210 (2012) 027.

[31] A. de Gouvea and S. Shalgar, Transition magnetic moments and collective neutrino oscillations: Three-flavor effects and detectability, JCAP 1304 (2013) 018.

[32] F. An et al. [JUNO Collaboration], Neutrino physics with JUNO, J. Phys. G 43 (2016) 030401.

[33] C. Giunti, K. Kouzakov, Y. F. Li, A. Lokhov, A. Studenikin, S. Zhou, Electromagnetic neutrinos in laboratory experiments and astrophysics, Annalen Phys. 528 (2016) 198

[34] J.S. Lu, Y.-F. Li and S. Zhou, Getting the most from the detection of Galactic supernova neutrinos in future large liquid-scintillator detectors, Phys. Rev. D 94 (2016) 023006. 